\title{
Markers of Sleep Disordered Breathing and Diabetes Mellitus in a Multiethnic Sample of US Adults: Results from the National Health and Nutrition Examination Survey (2005-2008)
}

\author{
Charumathi Sabanayagam, ${ }^{1,2}$ Srinivas Teppala, ${ }^{1}$ and Anoop Shankar ${ }^{1}$ \\ ${ }^{1}$ Department of Community Medicine, West Virginia University School of Medicine, Robert C. Byrd Health Sciences Center, \\ Medical Center Drive, P.O. Box 9190, Morgantown, WV 26505-9190, USA \\ ${ }^{2}$ Singapore Eye Research Institute, Singapore National Eye Center, 11 Third Hospital Avenue, No. 05-00, Singapore 168751
}

Correspondence should be addressed to Anoop Shankar, ashankar@hsc.wvu.edu

Received 31 May 2011; Revised 23 September 2011; Accepted 25 October 2011

Academic Editor: D. S. Jessop

Copyright (C) 2012 Charumathi Sabanayagam et al. This is an open access article distributed under the Creative Commons Attribution License, which permits unrestricted use, distribution, and reproduction in any medium, provided the original work is properly cited.

\begin{abstract}
We examined gender and ethnic differences in the association between sleep disordered breathing (SDB) and diabetes among 6,522 participants aged $\geq 20$ years from the National Health and Nutrition Examination Survey 2005-08. SDB severity was defined based on an additive summary score including sleep duration, snoring, snorting, and daytime sleepiness. We found that the summary SDB score was significantly associated with diabetes after adjusting for potential confounders in the whole population. Compared to those without any sleep disturbance, the multivariable odds ratio (OR) (95\% confidence interval (CI)) of diabetes among those with $\geq 3$ sleep disturbances was 2.04 (1.46-2.87). In sex-specific analyses, this association was significant only in women (OR ( $95 \%$ $\mathrm{CI})=3.68(2.01-6.72))$ but not in men $(1.10(0.59-2.04)), P$-interaction $=0.01$. However, there were no ethnic differences in this association, $P$-interaction $=0.7$. In a nationally representative sample of US adults, SDB was independently associated with diabetes only in women, but not in men.
\end{abstract}

\section{Introduction}

Sleep disordered breathing (SDB) and sleep apnea are associated with cardiovascular disease [1] and mortality [2]. It has been estimated that approximately $12-18$ million adults are affected by SDB in the USA [3]. Recent studies conducted in the USA [4-10] and Europe [11-14] have shown an association between a variety of markers of SDB including snoring $[4,11,15]$, daytime sleepiness $[8,12,13]$, or sleep duration $[5-7,10,14]$ and diabetes. However, some of these studies used either a single marker of SDB or were conducted in specific populations including men [6], occupational cohorts [4], or older adults [9].

It is not clear if there are gender or ethnic differences in the association between SDB and diabetes in the USA. SDB is often underevaluated and underdiagnosed in women [16]. Previous studies, mostly conducted in the Europe $[9,12,13,17]$, have reported gender differences in the association between SDB and diabetes. Two of these studies have reported an association only in women $[12,17]$ while one study has reported an association in only men [13]. In the USA, Enright et al. reported that snoring was associated with diabetes in older women but not in older men in the Cardiovascular Health Study [9]. African Americans and Hispanic Americans were shown to be disproportionately affected by both sleep disturbances [18] and diabetes [19]. In studies involving predominantly white samples, including the Sleep Heart Health Study (93\% whites) [20] and the Wisconsin Sleep Cohort Study (96\% whites) [21], SDB was found to be associated with diabetes, whereas SDB was not associated with diabetes in a predominantly African American and Hispanic population in a clinical study in Chicago [22]. In this context, we examined the association between several subjective markers of SDB including snoring, snorting, daytime sleepiness, and sleep duration and diabetes in a nationally representative sample of US adults after accounting for depression and other potential confounders. We also examined the association between 
markers of SDB and diabetes stratified by gender and raceethnicity.

\section{Methods}

The data for this study is derived from the National Health and Nutrition Examination Survey (NHANES) 2005-2006 and 2007-2008. Detailed description of NHANES study design and methods is available elsewhere [23, 24]. In brief, the NHANES survey included a stratified multistage probability sample representative of the civilian noninstitutionalized US population. Selection was based on counties, blocks, households, and individuals within households, and included oversampling of non-Hispanic blacks and Mexican Americans in order to provide stable estimates of these groups. We restricted our study sample to participants aged greater than 20 years (age range 20-85 years). Questions on sleep were first included in the NHANES in 200506. Of the 10,914 participants with information on sleep variables, after excluding those with pregnancy $(n=393)$, prevalent cardiovascular disease $(n=1,275)$, those with missing information on fasting or nonfasting glucose or sleep variables $(n=2,109)$, and those included in the multivariable model $(n=615), 6,522$ were available for the final analysis.

2.1. Outcome of Interest: Diabetes Mellitus. Diabetes was defined as a serum glucose $\geq 126 \mathrm{mg} / \mathrm{dL}$ after fasting for a minimum of 8 hours, a plasma glucose $\geq 200 \mathrm{mg} / \mathrm{dL}$ for those who fasted $<8$ hours before their NHANES visit, or glycosylated hemoglobin (HbAlc) $\geq 6.5 \%$, a selfreported physician-diagnosed diabetes or current use of oral hypoglycemic medication or insulin [25]. Because NHANES does not collect information (e.g., c-peptide levels) to identify the type of diabetes, we did not distinguish between type 1 and type 2 diabetes. However, we believe that the majority of diabetes subjects in our sample are due to type 2 diabetes mellitus. Fasting plasma glucose levels were measured using hexokinase enzymatic method on Roche/Hitachi Modular P Chemistry Analyzer at the Fairview Medical Center Laboratory at the University of Minnesota, Minneapolis MN. Random plasma glucose levels were measured at the Collaborative Laboratory Services in Ottumwa, IA using the Beckman Synchron LX20 in 2007 and the Beckman Coulter UniCel DxC800 in 2008. Glycosylated hemoglobin measurements were performed on the Tosoh 2.2 Analyzer (Tosoh Medics, Inc., 347 Oyster Pt. Boulevard, Suite 201, So., San Francisco, Ca) in NHANES 2005-06 and on the Automated HPLC System Glycohemoglobin Analyzer (Tosoh Medics, Inc., 347 Oyster Pt. Blvd., Suite 201, So. San Francisco, Ca 94080) in NHANES 2007-08 at the University of Minnesota, Minneapolis Minnesota.

2.2. Assessment of Exposure. A questionnaire on sleep habits based on validated questions from previous epidemiological studies was introduced in NHANES from 2005 till 2008 [26]. SDB was assessed from a set of questions on sleep habits including "How much sleep do you usually get at night on weekdays or workdays?", "In the past 12 months, how often did you snore while you were sleeping?", "In the past 12 months, how often did you snort, gasp or stop breathing while you were asleep?", and "In the past month, how often did you feel excessively or overly sleepy during the day?" Based on the responses to the above questions, we created four sleep variables: sleep duration, snoring, snorting, and daytime sleepiness. Sleep duration coded in hours was categorized into $\leq 5,6,7,8$, and $\geq 9 \mathrm{~h}$. Snoring and snorting variables were categorized into never or rare, occasional (3-4 nights/week), and frequent (5 or more nights/week). Daytime sleepiness was categorized into never or rare, sometimes (2-4 times/month), and often or almost always (5 or more times/month).

Based on the rationale that subjects with multiple SDB markers (e.g., snoring, snorting, daytime sleepiness) are likely to have more severe SDB, we developed an additive SDB summary score to examine the effect of cooccurrence of various SDB markers in relation to diabetes mellitus. For the summary score, we first dichotomized the individual variables based on their clinical significance and previous literature $[10,27,28]$. A score of 1 was assigned separately if the participants report sleep duration of $\leq 5$, snoring at least 3-4 nights/week, snorting at least 3-4 nights/week and daytime sleepiness at least 5 times/month. The summary score ranged from 0 to 4 corresponding to no sleep disturbance to coexistence of all 4 sleep disturbances. We assessed diagnosed sleep apnea from the question, "Have you ever been told by a doctor or other health professional that you have a sleep disorder?" with dichotomous responses ("yes" or "no").

2.3. Assessment of Covariates. Information on age, gender, race/ethnicity, smoking status, alcohol intake (g/day), level of education, history of diabetes and oral hypoglycemic intake or insulin administration, and antihypertensive medication use was obtained during a standardized questionnaire at home interview. Educational attainment was categorized into less than high school graduate, high school graduate, and more than high school graduate. Individuals who had smoked $<100$ cigarettes during their lifetime were considered never smokers, those who had smoked $\geq 100$ cigarettes lifetime and currently not smoking were considered former smokers, and those who had smoked $\geq 100$ cigarettes lifetime and currently smoking were considered current smokers. Current alcohol drinking was defined as consumption of $\geq 1$ alcoholic drink in the past 12 months. Moderate physical activity was defined as engaging in moderate-intensity sports, fitness, or recreational activities that cause a small increase in breathing or heart rate such as brisk walking, bicycling, swimming, or golf for at least 10 minutes continuously in a typical week. Information on anthropometric, physical, and laboratory components was obtained during the medical examination center (MEC) examination. Body weight measured in pounds using a digital scale was converted to kilograms. Standing height was measured using a stadiometer and corrected to the nearest tenth of a centimeter [29]. Body mass index (BMI) was calculated as weight in kilograms divided by heights in meter squared. We defined overweight as BMI $\geq 25 \mathrm{~kg} / \mathrm{m}^{2}$ and 
obesity as BMI $\geq 30 \mathrm{~kg} / \mathrm{m}^{2}$ based on the WHO international classification [30] and the US Centers for Disease Control and Prevention guidelines [31]. Blood pressure (BP) was measured using a mercury sphygmomanometer, and an average of three measurements was taken as the systolic and diastolic BP value. Hypertension was defined as a systolic $\mathrm{BP} \geq 140 \mathrm{~mm} \mathrm{Hg}$ or a diastolic BP $\geq 90 \mathrm{~mm} \mathrm{Hg}$ or a selfreported physician-diagnosed hypertension or current use of BP reducing medication [32]. Depression was assessed using the Patient Health Questionnaire (PHQ-9), a well-validated 9-item screening tool that asks questions about the frequency of symptoms of depression over the past 2 weeks [33]. Depression was defined as a PHQ-9 score of 10 or higher, a validated cut-point commonly used in clinical studies [33].

Detailed description about the blood collection, processing, and quality control checks are provided in the Laboratory Procedures Manual $[34,35]$. Serum C-reactive protein (CRP) was measured using latex-enhanced nephelometry. Total serum cholesterol was measured enzymatically using the Roche Hitachi 717 in 2005, Roche Hitachi 717 and 912 in 2006, and Roche Modular P chemistry analyzer in 20072008.

2.4. Statistical Analysis. We compared the characteristics of the study participants by diabetes status employing the chi square-test or analysis of variance as appropriate. We chose sleep duration of 7 hours as the reference category as previous studies have shown 7 hours of sleep to be associated with lower risk of CVD and mortality [36, 37]. We examined the association between categories of individual sleep variables, including snoring, snorting, daytime sleepiness, and sleep duration and diabetes in two multivariable models. In the first model, we adjusted for age (years) and sex (female, male). In the second model, we additionally adjusted for race-ethnicity (non-Hispanic whites, non-Hispanic blacks, Hispanic Americans, others), education ( $<$ high school, high school, > high school), smoking (never, former, current), current alcohol consumption (absent, present), physical activity (absent, present), body mass index $\left(\mathrm{kg} / \mathrm{m}^{2}\right)$, systolic blood pressure $(\mathrm{mm} \mathrm{Hg})$, depression (absent, present), CRP $(\mathrm{mg} / \mathrm{dL})$, and total cholesterol $(\mathrm{mg} / \mathrm{dL})$.

To examine the overall effect of SDB markers on diabetes, we created an additive summary SDB score and examined the association between this summary SDB score and diabetes in separate analyses. Tests for trend were performed using the categories of individual sleep variables and the summary score as an ordinal variable in the corresponding multivariable models. We tested for interactions between the summary SDB score and sex, race-ethnicity, and BMI categories by including cross-product interaction terms in the second multivariable model. To examine the consistency of the association we performed subgroup analyses stratified by sex, race-ethnicity, and BMI categories. All analyses were weighted to account for the unequal probabilities of selection, oversampling, and nonresponse using SUDAAN (version 8.0; Research Triangle Institute, Research Triangle Park, NC) and SAS (version 9.1.; SAS Institute, Cary, NC) software. In a supplementary analysis, we examined the association between diagnosed sleep apnea and diabetes.

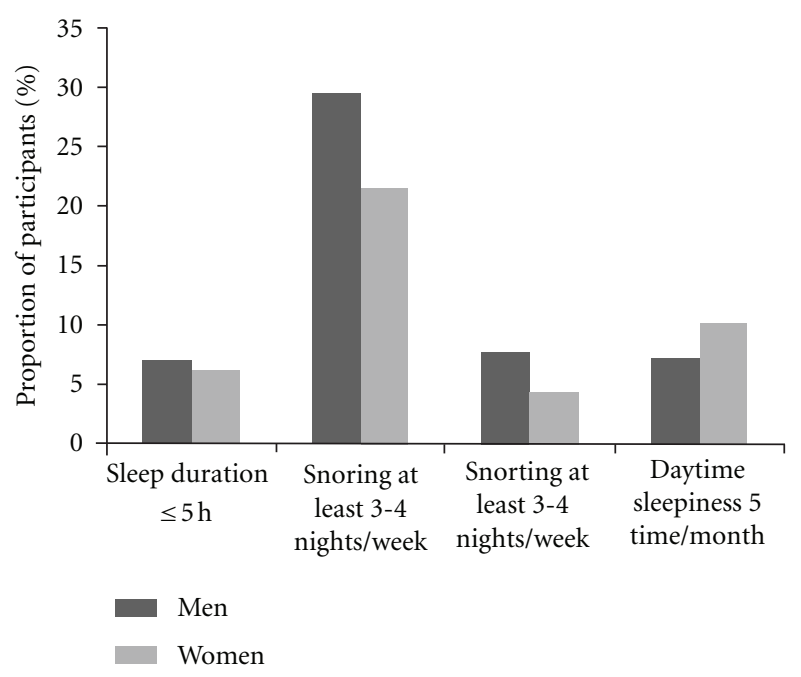

Figure 1: Proportion of participants by gender and by sleep disturbances.

\section{Results}

The prevalence of diabetes in the study population was $9 \%$. Table 1 shows the characteristics of the study population by diabetes status. Those with diabetes were more likely to be older, obese, had higher prevalence of hypertension, depression and higher levels of CRP, less likely to be non-Hispanic whites, above high school educated, current smokers, current drinkers, physically active, and had lower levels of total cholesterol.

Figure 1 shows the proportion of participants reporting sleep disturbances among men and women. Men were more likely to report snorting or snorting at least 3-4 nights/week (both $P<0.0001$ ), and women were more likely to report daytime sleepiness at least 5 times/month $(P=0.0002)$.

Table 2 shows the association between the various sleep variables and diabetes mellitus. Compared to sleep duration of $7 \mathrm{~h}$ (referent), sleep duration $\leq 5 \mathrm{~h}$ was positively associated with diabetes in both the age-, sex-adjusted and the multivariable models. Occasional or frequent snoring (at least 3-4 nights/week), frequent snorting (at least 5 times/week), and frequent daytime sleepiness (at least 5 times/month) were significantly associated with diabetes in both the age-, sex-adjusted and the multivariable models. A significant graded association was observed between increasing number of sleep disturbances and diabetes $(P$ trend $<0.0001$ ).

Table 3 shows the association between the summary SDB score and diabetes stratified by race-ethnicity. Consistent with the main results in Table 2, the association between summary SDB score and diabetes was found to be consistently present in all race-ethnic groups $(P$-interaction between SDB score and race-ethnicity $=0.7$ ). Table 4 shows the association between the summary SDB score and diabetes stratified by gender. The association between the summary SDB score and diabetes was significant in both men and women in the age-adjusted model, but in the 
TABLE 1: Baseline characteristics of the study population*.

\begin{tabular}{|c|c|c|c|}
\hline Characteristics & Diabetes absent $(n=5685)$ & Diabetes present $(n=837)$ & $P$-value \\
\hline Women (\%) & $2782(50.6)$ & $398(50.1)$ & 0.454 \\
\hline Age (years) & $43.7 \pm 0.4$ & $55.4 \pm 0.6$ & $<0.0001$ \\
\hline Race/Ethnicity (\%) & & & 0.0002 \\
\hline Non-Hispanic Whites & $2898(73.5)$ & $321(63.0)$ & \\
\hline Non-Hispanic Blacks & $1085(9.4)$ & $238(16.8)$ & \\
\hline Mexican Americans & $1021(7.8)$ & $185(9.7)$ & \\
\hline Others & $681(9.3)$ & $93(10.4)$ & \\
\hline Education categories (\%) & & & $<0.0001$ \\
\hline Below high school & $1409(16.1)$ & $335(24.8)$ & \\
\hline High school & $1391(24.3)$ & $197(26.5)$ & \\
\hline Above high school & $2885(59.5)$ & $305(48.7)$ & \\
\hline Smoking (\%) & & & 0.184 \\
\hline Never smoker & $3045(52.9)$ & $428(52.1)$ & \\
\hline Former smoker & $1284(22.9)$ & $263(31.0)$ & \\
\hline Current smoker & $1356(24.2)$ & $146(16.9)$ & \\
\hline Current drinker (\%) & $4056(76.3)$ & $406(54.0)$ & $<0.0001$ \\
\hline Moderate physical activity (\%) & $2741(54.9)$ & $286(39.0)$ & $<0.0001$ \\
\hline Body mass index $(\%)$ & & & $<0.0001$ \\
\hline Normal weight & $1869(35.2)$ & $114(12.1)$ & \\
\hline Overweight & $2036(34.6)$ & $242(26.3)$ & \\
\hline Obese & $1780(30.1)$ & $481(61.6)$ & \\
\hline Depression (\%) & $207(3.1)$ & $52(6.4)$ & 0.002 \\
\hline Systolic blood pressure ( $\mathrm{mm} \mathrm{Hg}$ ) & $119.7 \pm 0.3$ & $129.3 \pm 0.8$ & $<0.0001$ \\
\hline C-reactive protein $(\mathrm{mg} / \mathrm{dL})$ & $0.4 \pm 0.01$ & $0.6 \pm 0.04$ & $<0.0001$ \\
\hline Total cholesterol (mg/dL) & $200.7 \pm 0.8$ & $194.2 \pm 2.1$ & 0.03 \\
\hline
\end{tabular}

${ }^{*}$ Data presented are number (percentages) or mean values \pm standard error (SE), as appropriate for the variable.

multivariable model it was significant in women whereas it was substantially attenuated and not significant in men $(P$ interaction between SDB score and sex $=0.01$ ). In a subgroup analysis stratified by BMI categories, the association between summary sleep score and diabetes was consistently present among those with BMI $<25$ and $\geq 25 \mathrm{~kg} / \mathrm{m}^{2}$. The multivariable odds ratio (OR) (95\% confidence interval (CI)) of diabetes associated with SDB summary score was 1.36 (1.05-1.77) for BMI $<25 \mathrm{~kg} / \mathrm{m}^{2}$ and 1.21 (1.09-1.34) for $\mathrm{BMI} \geq 25 \mathrm{~kg} / \mathrm{m}^{2}$ ( $P$-interaction between SDB score and BMI categories $=0.2)($ data not shown $)$.

In a supplementary analysis, we examined the association between diagnosed sleep apnea and diabetes. $4.3 \%$ of the participants reported having diagnosed sleep apnea. Similar to the individual sleep variables, a significant positive association was observed between diagnosed sleep apnea and diabetes. Compared to those without diagnosed sleep apnea, the multivariable odds ratio (OR) (95\% confidence interval (CI)) of diabetes among those with diagnosed sleep apnea was 1.85 (1.19-2.89).

\section{Discussion}

In a representative sample of US adults, we found that markers of SDB including short sleep duration $(\leq 5 \mathrm{~h})$, occasional or frequent snoring (at least 3-4 nights/week), frequent snorting (at least 5 times/week), and frequent daytime sleepiness (at least 5 times/month) were associated with diabetes mellitus independent of age, sex, race-ethnicity, education, smoking, current alcohol consumption, physical activity, BMI, systolic blood pressure, depression, CRP and total cholesterol. Further, an ad hoc summary SDB score that provided a measure of the severity of SDB by counting the cooccurrence of these four sleep disturbances showed that, compared to those without any SDB markers, those with three or more SDB markers had more than twofold odds of having diabetes. In subgroup analyses, there were no differences in this association by race-ethnicity or by BMI categories. However, the association between the summary SDB score and diabetes was significant only in women; the association was substantially attenuated and lost statistical significance in men after multivariable adjustment for lifestyle and metabolic risk factors.

In the current study, we found that SDB, defined by short sleep duration, occasional or frequent snoring, frequent snorting, and frequent daytime sleepiness was associated with diabetes consistent with previous studies $[4,5,7,8$, $10,11,15]$. In the Sleep in America poll, daytime sleepiness but not snoring, snorting, and short sleep duration were associated with diabetes among 1506 men and women [8]. Both short and long durations of sleep were shown to be associated with diabetes among men in the Massachusetts 
TABLE 2: Association between sleep variables and diabetes mellitus.

\begin{tabular}{|c|c|c|c|c|}
\hline Sleep variables & $\begin{array}{l}\text { No. at risk } \\
(n=6522) \\
\end{array}$ & $\begin{array}{l}\text { Diabetes cases } \\
\quad(n=837) \\
\end{array}$ & $\begin{array}{l}\text { Age-, sex-adjusted odds } \\
\text { ratio }(95 \% \mathrm{CI})\end{array}$ & $\begin{array}{l}\text { Multivariable-adjusted } \\
\text { odds ratio* (95\% CI) }\end{array}$ \\
\hline \multicolumn{5}{|l|}{ Sleep duration (hours) } \\
\hline$\leq 5 \mathrm{hrs}$ & 1008 & 168 & $1.91(1.42-2.58)$ & $1.38(1.01-1.90)$ \\
\hline $6 \mathrm{hrs}$ & 1509 & 177 & $1.16(0.89-1.53)$ & $1.12(0.80-1.56)$ \\
\hline $7 \mathrm{hrs}$ & 1877 & 201 & 1 (referent) & 1 (referent) \\
\hline $8 \mathrm{hrs}$ & 1719 & 234 & $1.09(0.83-1.43)$ & $1.12(0.83-1.51)$ \\
\hline$\geq 9 \mathrm{hrs}$ & 409 & 57 & $0.91(0.55-1.51)$ & $0.79(0.47-1.34)$ \\
\hline$P$-trend & & & 0.0001 & 0.04 \\
\hline \multicolumn{5}{|l|}{ Frequency of snoring } \\
\hline Never or rarely & 3158 & 298 & 1 (referent) & 1 (referent) \\
\hline Occasionally & 1256 & 184 & $1.93(1.44-2.59)$ & $1.67(1.23-2.27)$ \\
\hline Frequently & 2108 & 355 & $1.94(1.58-2.39)$ & $1.44(1.16-1.79)$ \\
\hline$P$-trend & & & $<0.0001$ & 0.0008 \\
\hline \multicolumn{5}{|l|}{ Frequency of snorting } \\
\hline Never or rarely & 5718 & 684 & 1 (referent) & 1 (referent) \\
\hline Occasionally & 421 & 80 & $1.23(0.87-1.73)$ & $0.94(0.65-1.36)$ \\
\hline Frequently & 383 & 73 & $1.98(1.48-2.66)$ & $1.37(1.04-1.79)$ \\
\hline$P$-trend & & & $<0.0001$ & 0.09 \\
\hline \multicolumn{5}{|l|}{$\begin{array}{l}\text { Frequency of daytime } \\
\text { sleepiness }\end{array}$} \\
\hline Never or rarely & 3768 & 481 & 1 (referent) & 1 (referent) \\
\hline Sometimes & 1680 & 198 & $1.02(0.86-1.22)$ & $1.01(0.81-1.25)$ \\
\hline Often and almost always & 1074 & 158 & $1.51(1.25-1.83)$ & $1.38(1.08-1.76)$ \\
\hline$P$-trend & & & 0.0002 & 0.03 \\
\hline \multicolumn{5}{|l|}{ SDB summary score } \\
\hline 0 & 2353 & 207 & 1 (referent) & 1 (referent) \\
\hline 1 & 2573 & 345 & $1.64(1.25-2.16)$ & $1.37(1.04-1.80)$ \\
\hline 2 & 1177 & 203 & $2.03(1.60-2.57)$ & $1.45(1.14-1.85)$ \\
\hline$\geq 3$ & 419 & 82 & $3.50(2.55-4.81)$ & $2.04(1.46-2.87)$ \\
\hline$P$-trend & & & $<0.0001$ & $<0.0001$ \\
\hline
\end{tabular}

* Adjusted for age (years), sex (men, women), race-ethnicity (non-Hispanic whites, non-Hispanic blacks, Mexican Americans, others), education (< high school, high school, > high school), smoking (never, former, current), current alcohol consumption (absent, present), moderate physical activity (times/week), body mass index $\left(\mathrm{kg} / \mathrm{m}^{2}\right)$, depression (absent, present), systolic blood pressure $(\mathrm{mm} \mathrm{Hg})$, C-reactive protein $(\mathrm{mg} / \mathrm{dL})$ and total cholesterol ( $\mathrm{mg} / \mathrm{dL}$ ).

Male Aging Study [6], among participants in the first National Health and Nutrition Examination Survey Followup Study [10], among a large cohort of participants in the National Health Interview Survey 2004-2005 [7], and men and women in the Sleep Heart Health Study [5]. In contrast, in the Finnish Diabetes Prevention Study, long sleep duration was associated with diabetes among overweight individuals with impaired glucose tolerance [14]. Self-reported snoring was associated with 10-year incidence of diabetes in a large cohort of nurses in the USA [4]. Several studies conducted in the Europe have documented similar associations between self-reported short sleep duration [38], snoring [11, 15], and daytime sleepiness [15] and diabetes. Sleep duration $\leq 5 \mathrm{~h}$ was associated with 12-year incidence of diabetes in Swedish men but not in women [38]. Snoring was associated with 10year incidence of self-reported diabetes in 2668 men aged 3069 years in Sweden. Further, joint exposure to both snoring and obesity had a fivefold odds of diabetes compared with their absence [11]. In a cross-sectional study in Finland, selfreported snoring but not daytime sleepiness was associated with diabetes among 593 men and women [15].

Our study contributes to the existing literature on SDB by demonstrating, in detailed subgroup analyses, that the SDB-diabetes association (1) was consistently present in both non-Hispanic whites and other race-ethnicities, but that (2) there were sex-related differences in this association. First, to our knowledge, this is the first population-based study that compared the association between SDB and diabetes side by side by race-ethnicity as previous studies were conducted either in predominantly white samples or samples involving only African Americans and Hispanic Americans.

Second, in the subgroup analysis by sex, we found that even though there was a positive association between SDB markers and diabetes in both sexes in the initial ageadjusted model, upon multivariable adjustment for lifestyle and metabolic risk factors, the association was no longer 
TABLE 3: Association between SDB and diabetes mellitus, by race/ethnicity.

\begin{tabular}{lcccc}
\hline SDB summary score & No. at risk & Diabetes cases & Age-, sex-adjusted odds ratio (95\% CI) & Multivariable-adjusted odds ratio* (95\% CI) \\
\hline Non-Hispanic Whites & & & & 1 (referent) \\
0 & 1209 & 84 & 1 (referent) & $1.42(0.94-2.14)$ \\
1 & 1242 & 131 & $1.73(1.17-2.55)$ & $1.48(1.06-2.07)$ \\
2 & 566 & 74 & $2.12(1.48-3.03)$ & $2.21(1.42-3.45)$ \\
$\geq 3$ & 202 & 32 & $3.94(2.40-6.48)$ & $<0.0001$ \\
$P$-trend & & & $<0.0001$ & 1 (referent) \\
Other race-ethnicities & & & & $1.25(0.91-1.70)$ \\
0 & 1144 & 123 & $1.40(1.07-1.83)$ & $1.38(0.91-2.10)$ \\
1 & 1331 & 214 & $1.75(1.22-2.51)$ & $1.73(0.98-3.06)$ \\
2 & 611 & 129 & $2.46(1.52-3.99)$ & 0.03 \\
$\geq 3$ & 217 & 50 & $<0.0001$ & \\
$P$-trend & & &
\end{tabular}

${ }^{*}$ Adjusted for age (years), sex (men, women), education (< high school, high school, $>$ high school), smoking (never, former, current), current alcohol consumption (absent, present), moderate physical activity (times/week), body mass index $\left(\mathrm{kg} / \mathrm{m}^{2}\right)$, depression (absent, present), systolic blood pressure $(\mathrm{mm} \mathrm{Hg})$, C-reactive protein $(\mathrm{mg} / \mathrm{dL})$, and total cholesterol $(\mathrm{mg} / \mathrm{dL})$.

$P$-interaction $(\mathrm{SDB}$ summary score $\times$ race-ethnicity $)=0.7$.

TABLE 4: Association between SDB and diabetes mellitus, by gender.

\begin{tabular}{|c|c|c|c|c|}
\hline SDB summary score & No. at risk & Diabetes cases & Age-adjusted odds ratio $(95 \% \mathrm{CI})$ & Multivariable-adjusted odds ratio* $(95 \% \mathrm{CI})$ \\
\hline \multicolumn{5}{|l|}{ Men } \\
\hline 0 & 1065 & 109 & 1 (referent) & 1 (referent) \\
\hline 1 & 1360 & 180 & $1.56(1.17-2.09)$ & $1.38(1.03-1.86)$ \\
\hline 2 & 683 & 114 & $1.91(1.32-2.76)$ & $1.42(0.96-2.09)$ \\
\hline$\geq 3$ & 234 & 36 & $1.82(1.12-2.94)$ & $1.10(0.59-2.04)$ \\
\hline$P$-trend & & & 0.0001 & 0.25 \\
\hline \multicolumn{5}{|l|}{ Women } \\
\hline 0 & 1288 & 98 & 1 (referent) & 1 (referent) \\
\hline 1 & 1213 & 165 & $1.68(1.17-2.43)$ & $1.31(0.87-2.00)$ \\
\hline 2 & 494 & 89 & $2.06(1.51-2.82)$ & $1.48(0.99-2.20)$ \\
\hline$\geq 3$ & 185 & 46 & $6.03(3.65-9.96)$ & $3.68(2.01-6.72)$ \\
\hline$P$-trend & & & $<0.0001$ & $<0.0001$ \\
\hline
\end{tabular}

${ }^{*}$ Adjusted for age (years), race-ethnicity (non-Hispanic whites, non-Hispanic blacks, Mexican Americans, others), education (< high school, high school, $>$ high school), smoking (never, former, current), current alcohol consumption (absent, present), moderate physical activity (times/week), body mass index $\left(\mathrm{kg} / \mathrm{m}^{2}\right)$, depression (absent, present), systolic blood pressure $(\mathrm{mm} \mathrm{Hg})$, C-reactive protein $(\mathrm{mg} / \mathrm{dL})$, and total cholesterol $(\mathrm{mg} / \mathrm{dL})$.

$P$-interaction $($ SDB summary score $\mathrm{f} \times$ female $)=0.01$.

present in men whereas strongly present in women. This finding indirectly suggests that the role of SDB in men is explained by lifestyle and metabolic risk factors such as BMI, lack of physical activity, depression, high CRP, and others where as in women SDB has a residual association that is independent of these factors. Finally, our finding of an independent association in women but not in men is consistent with some $[9,17,39]$ but not all previous studies [13]. For example, in the Cardiovascular Health Study, involving 5201 men and women aged $\geq 65$ years in the USA, snoring was associated with diabetes in older women but not in older men [9]. In the MONICA study involving 7905 men and women, snoring was associated with diabetes only in women [17]. In a clinic-based study involving 318 men and women aged 30-69 years in Sweden, SDB defined by polysomnographic measured overnight oxygen desaturation was associated with diabetes in women but not in men [39].
In contrast, in a cross-sectional study involving 1396 men and 1500 women aged 45-74 years in Finland, self-reported SDB was associated with diabetes in middle-aged men but not in women [13].

Several mechanisms have been proposed to explain the association between SDB and diabetes. Epidemiological studies have shown that short sleep duration is associated with altered glucose metabolism [40], poor glycemic control [41], insulin resistance [40], and markers of inflammation [42]. Snoring with resultant intermittent hypoxia leads to insulin resistance and diabetes [43] through a variety of mechanisms, including activation of the sympathetic nervous system resulting in elevated catecholamine levels [44], activation of the hypothalamic-pituitary-adrenal axis resulting in elevated cortisol levels [45], formation of reactive oxygen species and increased oxidative stress [46], and activation of proinflammatory cytokines [47]. 
The large multiethnic sample with rich information on potential confounders and the rigorous methodology of data collection in NHANES are the main strengths of the study. Our study has some limitations. First, assessment of SDB from self-reported sleep measures might have resulted in nondifferential misclassification biasing the association towards null. Second, even though we adjusted for obesity in the multivariable analysis and also performed stratified analysis by BMI categories, it is possible that there is residual confounding by obesity status which may be biasing our results. Third, it is possible that some of the markers that we use to define SDB are biased. For example, there may be gender and/or racial differences in the self-report of snoring. Without polysomnographic examination, it is difficult to avoid measurement error due to self-report. Fourth, our findings are generalizable only to the US noninstitutionalized civilian population; the results may not be representative of institutionalized people or populations outside the USA. Fifth, our cross-sectional study design limits making conclusion on the temporal associations between SDB and diabetes.

In conclusion, in a nationally representative sample of US adults, we found that sleep duration $\leq 5 \mathrm{~h}$, occasional or frequent snoring, frequent snorting, and frequent daytime sleepiness were associated with diabetes mellitus independent of potential confounders. This association was significant only in women but consistently present in all ethnic groups.

\section{Conflict of Interests}

There is no conflict of interest related to this paper.

\section{Disclosure}

"The guarantor, C. Sabanayagam, accepts full responsibility for the work and/or the conduct of the study, had access to the data, and controlled the decision to publish".

\section{Acknowledgment}

This study was funded by an American Heart Association national Clinical Research program grant (A. Shankar).

\section{References}

[1] R. Budhiraja, P. Budhiraja, and S. F. Quan, "Sleep-disordered breathing and cardiovascular disorders," Respiratory Care, vol. 55, no. 10, pp. 1322-1330, 2010.

[2] N. M. Punjabi, B. S. Caffo, J. L. Goodwin et al., "Sleepdisordered breathing and mortality: a prospective cohort study," PLoS Medicine, vol. 6, no. 8, Article ID e1000132, 2009.

[3] T. Young, M. Palta, J. Dempsey, P. E. Peppard, F. J. Nieto, and K. M. Hla, "Burden of sleep apnea: rationale, design, and major findings of the Wisconsin sleep cohort study," Wisconsin Medical Journal, vol. 108, no. 5, pp. 246-249, 2009.

[4] W. K. Al-Delaimy, J. E. Manson, W. C. Willett, M. J. Stampfer, and F. B. Hu, "Snoring as a risk factor for type II diabetes mellitus: a prospective study," American Journal of Epidemiology, vol. 155, no. 5, pp. 387-393, 2002.
[5] D. J. Gottlieb, N. M. Punjabi, A. B. Newman et al., "Association of sleep time with diabetes mellitus and impaired glucose tolerance," Archives of Internal Medicine, vol. 165, no. 8, pp. 863-867, 2005.

[6] H. K. Yaggi, A. B. Araujo, and J. B. McKinlay, "Sleep duration as a risk factor for the development of type 2 diabetes," Diabetes Care, vol. 29, no. 3, pp. 657-661, 2006.

[7] O. M. Buxton and E. Marcelli, "Short and long sleep are positively associated with obesity, diabetes, hypertension, and cardiovascular disease among adults in the United States," Social Science and Medicine, vol. 71, no. 5, pp. 1027-1036, 2010.

[8] D. Foley, S. Ancoli-Israel, P. Britz, and J. Walsh, "Sleep disturbances and chronic disease in older adults: results of the 2003 National Sleep Foundation Sleep in America Survey," Journal of Psychosomatic Research, vol. 56, no. 5, pp. 497-502, 2004.

[9] P. L. Enright, A. B. Newman, P. W. Wahl, T. A. Manolio, E. F. Haponik, and P. J. Boyle, "Prevalence and correlates of snoring and observed apneas in 5,201 older adults," Sleep, vol. 19, no. 7, pp. 531-538, 1996.

[10] J. E. Gangwisch, S. B. Heymsfield, B. Boden-Albala et al., "Sleep duration as a risk factor for diabetes incidence in a large US sample," Sleep, vol. 30, no. 12, pp. 1667-1673, 2007.

[11] A. Elmasry, C. Janson, E. Lindberg, T. Gislason, M. A. Tageldin, and G. Boman, "The role of habitual snoring and obesity in the development of diabetes: a 10-year follow-up study in a male population," Journal of Internal Medicine, vol. 248, no. 1, pp. 13-20, 2000.

[12] E. Lindberg, C. Berne, K. A. Franklin, M. Svensson, and C. Janson, "Snoring and daytime sleepiness as risk factors for hypertension and diabetes in women-a population-based study," Respiratory Medicine, vol. 101, no. 6, pp. 1283-1290, 2007.

[13] H. Tuomilehto, M. Peltonen, M. Partinen et al., "Sleepdisordered breathing is related to an increased risk for type 2 diabetes in middle-aged men, but not in women -the FIND2D survey," Diabetes, Obesity and Metabolism, vol. 10, no. 6, pp. 468-475, 2008.

[14] H. Tuomilehto, M. Peltonen, M. Partinen et al., "Sleep duration, lifestyle intervention, and incidence of type 2 diabetes in impaired glucose tolerance: the finnish diabetes prevention study," Diabetes Care, vol. 32, no. 11, pp. 1965-1971, 2009.

[15] A. K. Renko, L. Hiltunen, M. Laakso, U. Rajala, and S. Keinänen-Kiukaanniemi, "The relationship of glucose tolerance to sleep disorders and daytime sleepiness," Diabetes Research and Clinical Practice, vol. 67, no. 1, pp. 84-91, 2005.

[16] T. Young, J. Skatrud, and P. E. Peppard, "Risk factors for obstructive sleep apnea in adults," Journal of the American Medical Association, vol. 291, no. 16, pp. 2013-2016, 2004.

[17] F. Valham, B. Stegmayr, M. Eriksson, E. Hägg, E. Lindberg, and K. A. Franklin, "Snoring and witnessed sleep apnea is related to diabetes mellitus in women," Sleep Medicine, vol. 10, no. 1, pp. 112-117, 2009.

[18] National Sleep Foundation, "Poll reveals differences among ethnic groups,” (2010), http://www.sleepfoundation.org/ press-releases/2010, accessed on March 09, 2011.

[19] National Diabetes Information Clearinghouse, "National Diabetes Statistics, 2011," http://diabetes.niddk.nih.gov/DM/ PUBS/statistics/\#Racial, accessed on March 9, 2011.

[20] N. M. Punjabi, E. Shahar, S. Redline, D. J. Gottlieb, R. Givelber, and H. E. Resnick, "Sleep-disordered breathing, glucose intolerance, and insulin resistance: the sleep heart health 
study," American Journal of Epidemiology, vol. 160, no. 6, pp. 521-530, 2004.

[21] K. J. Reichmuth, D. Austin, J. B. Skatrud, and T. Young, "Association of sleep apnea and type II diabetes: a populationbased study," American Journal of Respiratory and Critical Care Medicine, vol. 172, no. 12, pp. 1590-1595, 2005.

[22] K. Mahmood, N. Akhter, K. Eldeirawi et al., "Prevalence of type 2 diabetes in patients with obstructive sleep apnea in a multi-ethnic sample," Journal of Clinical Sleep Medicine, vol. 5, no. 3, pp. 215-221, 2009.

[23] National Center for Health Statistics, The National Health and Nutrition Examination Survey 2005-2006: survey Operations Manuals, Brochures, Consent Documents http://www.cdc .gov/nchs/nhanes/nhanes2005-2006/current_nhanes_05_06 .htm, Accessed Feb 25, 2011.

[24] National Center for Health Statistics, The National Health and Nutrition Examination Survey 2007-2008: survey Operations Manuals, Brochures, Consent Documents http://www.cdc .gov/nchs/nhanes/nhanes2007-2008/current_nhanes_07_08 .htm, Accessed Feb 25, 2011.

[25] Anonymous, "Standards of medical care in diabetes-2010," Diabetes Care, vol. 33, supplement 1, pp. S11-S61, 2010.

[26] H. R. Colten and B. M. Altevogt, Sleep Disorders and Sleep Deprivation: An Unmet Public Health Problem, National Academies Press, New York, NY, USA, Institue of Medicine (US) Committee on Sleep Medicine and Research, 2006.

[27] L. M. O’Brien, C. B. Mervis, C. R. Holbrook et al., "Neurobehavioral implications of habitual snoring in children," Pediatrics, vol. 114, no. 1, pp. 44-49, 2004.

[28] K. G. Baron, K. Liu, C. Chan, E. Shahar, R. HasnainWynia, and P. Zee, "Race and ethnic variation in excessive daytime sleepiness: the multi-ethnic study of atherosclerosis," Behavioral Sleep Medicine, vol. 8, no. 4, pp. 231-245, 2010.

[29] National Health and Nutrition Examination Survey, "Anthropometry and physical activity monitor procedures manual," http://www.cdc.gov/nchs/data/nhanes/nhanes_05_06/BM.pdf, accessed on 14 Sep 2011.

[30] Anonymous, "Obesity: preventing and managing the global epidemic. Report of a WHO consultation," Tech. Rep. 894, World Health Organization, Geneva, Switzerland, 2000.

[31] K. M. Flegal, M. D. Carroll, C. L. Ogden, and L. R. Curtin, "Prevalence and trends in obesity among US adults, 19992008," Journal of the American Medical Association, vol. 303, no. 3, pp. 235-241, 2010.

[32] A. V. Chobanian, G. L. Bakris, H. R. Black et al., "The seventh report of the joint National Committee on Prevention, detection, evaluation, and treatment of high blood pressure: the JNC 7 report," Journal of the American Medical Association, vol. 289, no. 19, pp. 2560-2572, 2003.

[33] K. Kroenke, R. L. Spitzer, and J. B. Williams, "The PHQ9: validity of a brief depression severity measure," Journal of General Internal Medicine, vol. 16, no. 9, pp. 606-613, 2001.

[34] National Center for Health Statistics, "Laboratory procedures used for NHANES 2005-2006," http://www.cdc.gov/nchs/, nhanes/nhanes2005-2006/lab05_06.htm, accessed Feb 25 2011.

[35] National Center for Health Statistics, "Laboratory procedures used for NHANES 2007-2008," http://www.cdc.gov/nchs/ nhanes/nhanes2007-2008/lab07_08.htm, accessed Feb 25 2011.

[36] A. Shankar, W. P. Koh, J. M. Yuan, H. P. Lee, and M. C. $\mathrm{Yu}$, "Sleep duration and coronary heart disease mortality among Chinese adults in Singapore: a population-based cohort study," American Journal of Epidemiology, vol. 168, no. 12, pp. 1367-1373, 2008.

[37] A. Tamakoshi and Y. Ohno, "Self-reported sleep duration as a predictor of all-cause mortality: results from the JACC Study, Japan," Sleep, vol. 27, no. 1, pp. 51-54, 2004.

[38] L. Mallon, J. E. Broman, and J. Hetta, "High incidence of diabetes in men with sleep complaints or short sleep duration: a 12-year follow-up study of a middle-aged population," Diabetes Care, vol. 28, no. 11, pp. 2762-2767, 2005.

[39] Y. T. Celen, J. Hedner, J. Carlson, and Y. Peker, "Impact of gender on incident diabetes mellitus in obstructive sleep apnea: a 16-year follow-up," Journal of Clinical Sleep Medicine, vol. 6, no. 3, pp. 244-250, 2010.

[40] L. Rafalson, R. P. Donahue, S. Stranges et al., "Short sleep duration is associated with the development of impaired fasting glucose: the western New York health study," Annals of Epidemiology, vol. 20, no. 12, pp. 883-889, 2010.

[41] K. L. Knutson, A. M. Ryden, B. A. Mander, and C. E. Van, "Role of sleep duration and quality in the risk and severity of type 2 diabetes mellitus," Archives of Internal Medicine, vol. 166, no. 16, pp. 1768-1774, 2006.

[42] S. R. Patel, X. Zhu, A. Storfer-Isser et al., "Sleep duration and biomarkers of inflammation," Sleep, vol. 32, no. 2, pp. 200204, 2009.

[43] J. E. Shaw, N. M. Punjabi, J. P. Wilding, K. G. Alberti, and P. Z. Zimmet, "Sleep-disordered breathing and type 2 diabetes: a report from the International Diabetes Federation Taskforce on Epidemiology and Prevention," Diabetes Research and Clinical Practice, vol. 81, no. 1, pp. 2-12, 2008.

[44] A. Xie, J. B. Skatrud, D. S. Puleo, and B. J. Morgan, "Exposure to hypoxia produces long-lasting sympathetic activation in humans," Journal of Applied Physiology, vol. 91, no. 4, pp. 1555-1562, 2001.

[45] M. Follenius, G. Brandenberger, J. J. Bandesapt, J. P. Libert, and J. Ehrhart, "Nocturnal cortisol release in relation to sleep structure," Sleep, vol. 15, no. 1, pp. 21-27, 1992.

[46] A. Bloch-Damti and N. Bashan, "Proposed mechanisms for the induction of insulin resistance by oxidative stress," Antioxidants and Redox Signaling, vol. 7, no. 11-12, pp. 15531567, 2005.

[47] A. Alberti, P. Sarchielli, E. Gallinella et al., "Plasma cytokine levels in patients with obstructive sleep apnea syndrome: a preliminary study," Journal of Sleep Research, vol. 12, no. 4, pp. 305-311, 2003. 


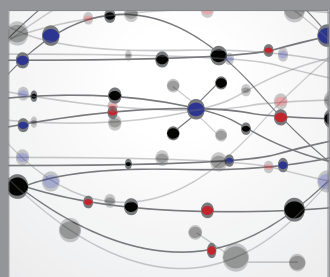

The Scientific World Journal
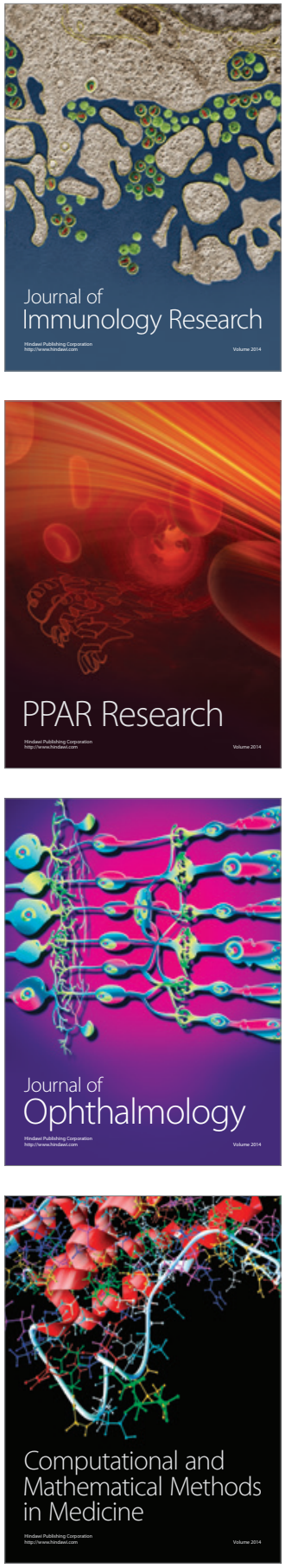

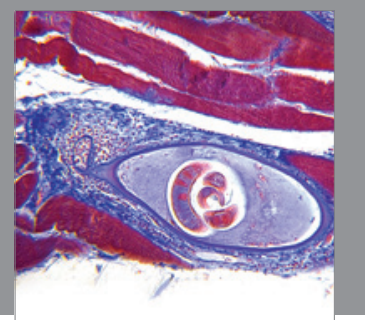

Gastroenterology

Research and Practice
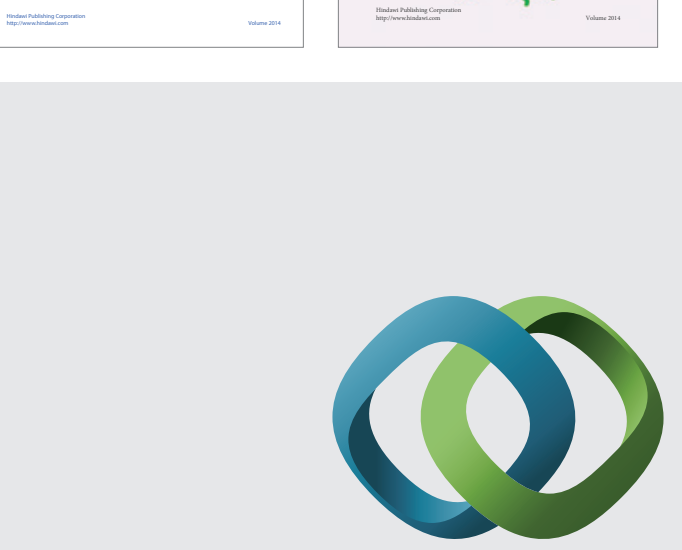

\section{Hindawi}

Submit your manuscripts at

http://www.hindawi.com
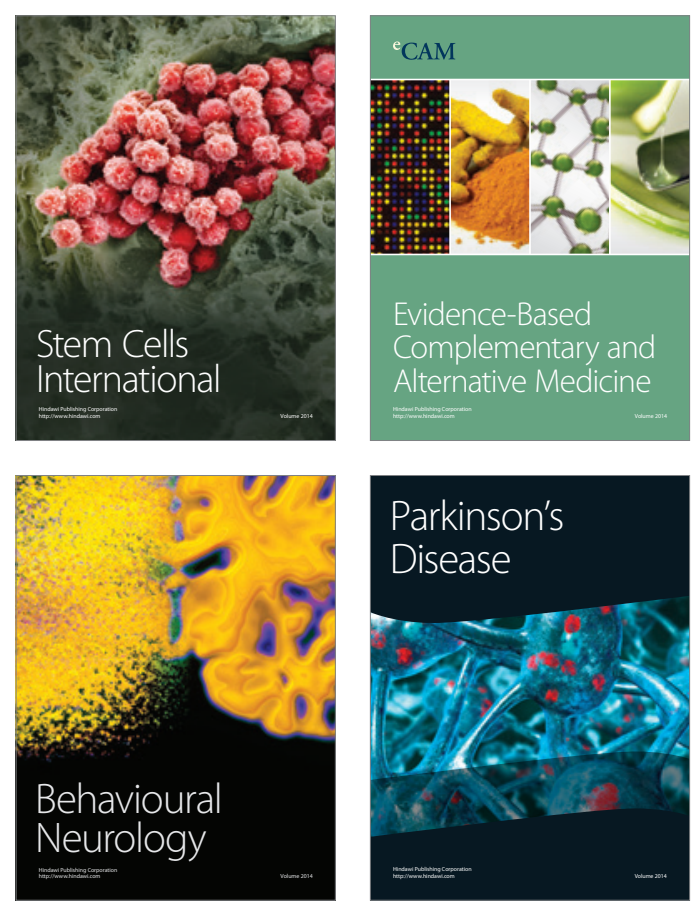

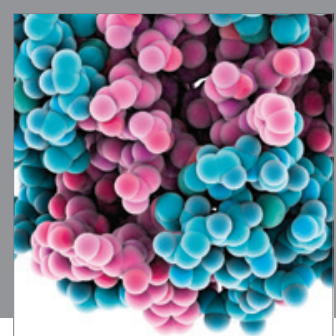

Journal of
Diabetes Research

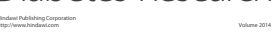

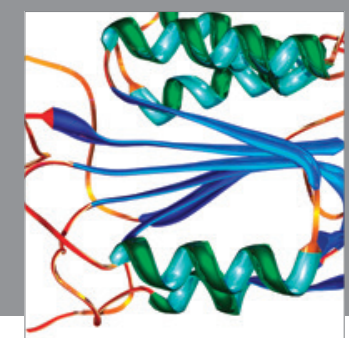

Disease Markers
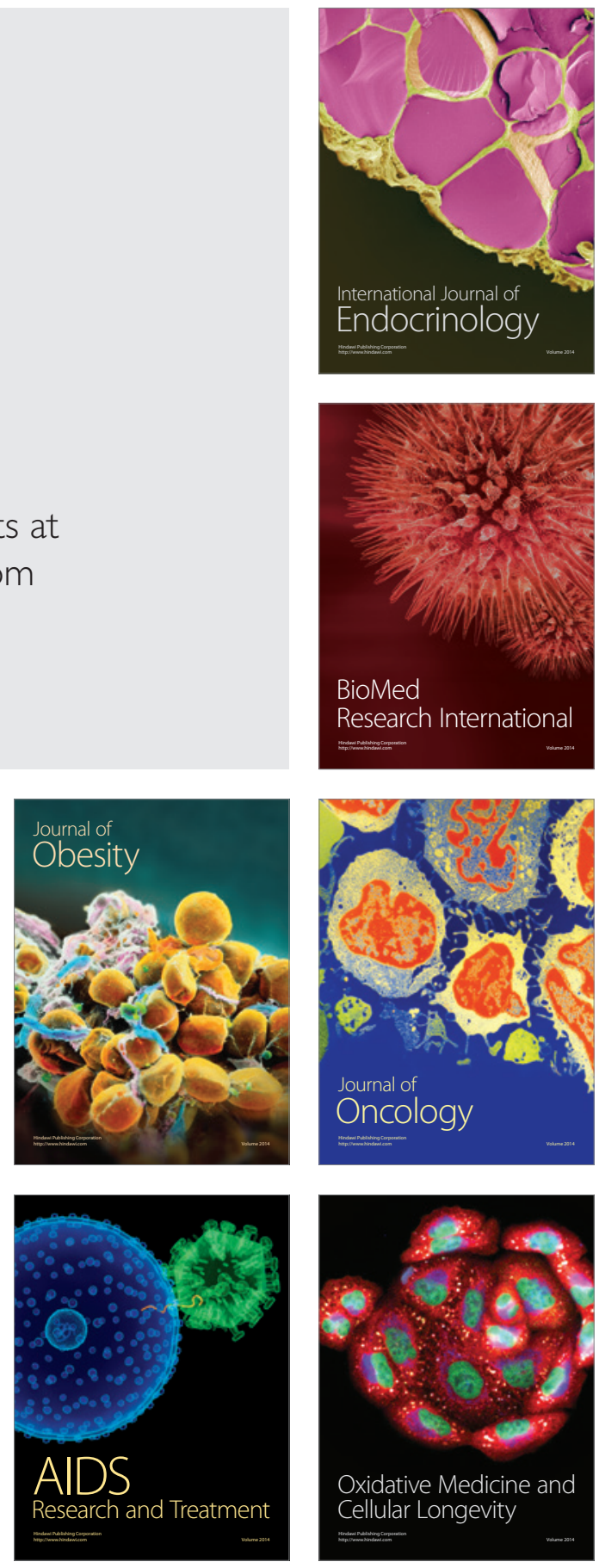